\title{
Rare Metachronous Multiple Carcinoma of Ear, Nose and Throat: A Case Report: Metachronous Multiple Carcinoma of Bilateral Maxillary Sinus and Lung
}

Yue Liu

Jilin University Second Hospital

Yan Liu

Jilin University Second Hospital

Qiu Jin Li

Jilin University Second Hospital

Xian Hai Guo

Jilin University Second Hospital

Xue Zhao

Jilin University Second Hospital

Dan Yu ( $\nabla$ yudanent@126.com ) second hospital of jilin university

\section{Case Report}

Keywords: multiple primary cancer (MPC), metachronous, maxillary sinus carcinoma, lung cancer, radiotherapy

Posted Date: November 19th, 2021

DOI: https://doi.org/10.21203/rs.3.rs-1078777/v1

License: (c) (i) This work is licensed under a Creative Commons Attribution 4.0 International License. Read Full License 


\section{Abstract}

\section{Backeground}

To report a rare case of metachronous multiple carcinoma of bilateral maxillary sinus and lung.

Methods

The clinical manifestations, pathological results, endoscopic findings and imaging features of the patient were collected and summarized.

Results

The patient, male, 58 years old, came to our department in 2010 for "left exophthalmos and blood in snot". Combined with relevant examinations, it was diagnosed as malignant tumor of the left maxillary sinus. Postoperative radiotherapy and chemotherapy were performed. Ten years later, the patient developed right maxillary sinus cancer and left lung cancer. The postoperative pathological return of lung was lung adenocarcinoma,excluding the possibility of metastasis.

\section{Conclusions}

For otorhinolaryngology,this case of isochronous multiple lung cancer in bilateral maxillary sinuses is very rare, which also suggests that we should closely follow up tumor patients in clinical treatment.In addition to the primary site, we should also strengthen the monitoring of radiotherapy operation area and easily metastatic organs (such as lung, bone, liver, etc.), strengthening the popularization of relevant knowledge and improve patients' awareness of disease prevention, changing the bad lifestyle, further reducing the incidence of multiple primary cancer or early detection of cancer and improving the quality of life of patients.

\section{Introduction}

Maxillary sinus cancer is a common malignant tumor in the head and neck, accounting for about $0.2 \%$ $3.0 \%$ of the malignant tumors in the whole body ${ }^{[1]}$. Among them, squamous cell carcinoma is the most common, and it is more common in patients in the middle and late stage of clinical practice. Due to the hidden anatomical site of maxillary sinus, complex surrounding relations and difficult early diagnosis, most patients have invaded the bone wall and surrounding tissues when they see a doctor, and the course of disease is mostly in the middle and late stage.At present, there is no unified treatment scheme, and the clinical cure rate of radiotherapy and chemotherapy alone is low. The reason is mainly related to local recurrence and tumor metastasis. Multiple primary cancer (MPC) refers to the occurrence of two or more primary malignant tumors in a single or multiple organs of the same host at the same time or at different times ${ }^{[2]}$. The incidence rate is not consistent at home and abroad. The incidence rate in the United States from 2005 to 2009 was $19 \%{ }^{[3]}$ and that in China was only $0.52 \% \sim 2.45 \%{ }^{[4]}$. Clinically, the misdiagnosis rate of multiple cancers is high, which is often misdiagnosed as cancer recurrence or 
metastasis and sometimes one of them is missed. Therefore, we should have enough understanding and vigilance. At present, there are few reports of metachronous multiple cancers in China, while it is more rare for the same patient to have different sides of maxillary sinus cancer, and the patient also has lung malignant tumors. By consulting relevant literature and reporting the characteristics of this case, we hope to enlighten the diagnosis and treatment of multiple lung cancer and reduce clinical misdiagnosis and mistreatment. The report is as follows:

\section{Case Presentation}

The patient, male, 58 years old, came to our department in $2010 \mathrm{for}$ "left exophthalmos and blood in the nose". The pathological results showed squamous cell carcinoma of the left maxillary sinus. Computed tomography(CT) and Magnetic resonance imaging(MRI)showed that the tumor destroyed the parietal and lateral walls of the left maxillary sinus (Fig. A-D). Combined with relevant examinations, he was diagnosed as malignant tumor of the left maxillary sinus. Because the tumor invaded the infraorbital wall, the patient requested eye protection treatment, Therefore, it is suggested that radiotherapy should be carried out first, and the dose should be up to $50 \mathrm{gy}$. We re evaluated the tumor before and after operation, and found that the tumor scope was significantly reduced. We underwent surgical treatment (resection of malignant tumor of left maxillary sinus and maxillary resection), and supplemented radiotherapy and chemotherapy after operation. The patients were closely followed up after systematic treatment. In 2019, there was persistent nasal obstruction on the right side, pain on the right face, radiation to the right upper dentition, and occasional blood in the nose. MRI of paranasal sinus showed abnormal signal shadows in right maxillary sinus, bilateral frontal sinus, ethmoid sinus and sphenoid sinus (Fig. E-G). Pathological examination of the right maxillary sinus showed squamous cell carcinoma infiltration in the tissue (right maxillary sinus). HRCT examination of the lung showed ground glass nodules in the lower lobe of the left lung (Fig.H). PET-CT examination showed that the lung was considered to be malignant. In order to exclude that the lung lesions were caused by maxillary cancer metastasis, wedge resection of lung and lobectomy with lymph node dissection were performed first. Postoperative pathological return: (left lower lobe) invasive adenocarcinoma (acinar type, invasion diameter about $0.6 \mathrm{~mm}$ ), surrounded by adenocarcinoma in situ, lesion about $1.5 \mathrm{~mm}$, no cancer invasion in vessels, a fibrous calcification junction in local areas, and no cancer in pleura and cutting edge. Immunohistochemical staining results: TTF-1 (+), napsina (+), p63 (scattered +), Ki67 (positive rate 10\%), CK5 / 6 (-), P40 (-). After lung surgery, the patient developed left pulmonary embolism, applied anticoagulants, and could not undergo surgical treatment. Therefore, radiotherapy was given to the right maxillary sinus cancer. After radiotherapy, the nasal sinus MRI (plain scan + enhancement) was rechecked: the tumor scope was significantly reduced, but there were still tumor residues in the anterior and inferior wall of the right maxillary sinus (Fig.I $-J$ ). Further particle implantation treatment (23 particles with 0.6 activity) was performed. After treatment, nasal endoscopy (Fig.K) and sinus MR (plain scan + enhancement) were rechecked: the tumor scope was reduced and the hard palate was broken (Fig.L). Considering that the tumor still shows progressive growth, radical surgery (resection of malignant tumor of right maxillary sinus + maxillary resection + repair of local pedicled temporal muscle flap (Fig.M)). Postoperative chemotherapy and PD-1 treatment 
were performed. At present, there is no tumor in the area of both maxillary sinuses. Reexamination of nasal sinus MRI(plain scan + enhancement): there is no abnormal signal shadow after operation in both maxillary sinuses (Fig.N). Eat well. So far, the tumor has no recurrence and is under follow-up.

\section{Discussion}

In recent years, with the improvement of the diagnosis and treatment of cancer, the long-term survival rate of malignant tumor patients has increased, and the incidence rate of multiple primary malignant tumors has been increasing. The diagnostic criteria of repeated cancer are as follows: (1) each tumor must be malignant histologically; (2) Each tumor has its unique pathological morphology; (3) The tumor occurred in different parts, and the two were not continuous; (4) The possibility of mutual transfer must be ruled out. The literature reports that about $14 \%$ of patients with malignant tumors may have new tumors, which is much higher than that of the normal population ${ }^{[5]}$. Respiratory system is a common site of multiple primary malignant tumors. The literature reports that the incidence of multiple primary tumors in patients with lung cancer is $0.2 \% \sim 1.8 \%{ }^{[6]}$. The cases of bilateral maxillary sinus cancer are rarely reported. In this paper, the patient has developed maxillary sinus cancer on different sides successively, which has the characteristics of heterochronism in the occurrence time of the tumor. It is very rare. At the same time, it is also complicated with lung malignant tumor, so it is more rare, which needs the attention of our otolaryngologists.

The shorter the tumor interval, the worse the prognosis. The reason may be that the time interval between two tumors is too short. When the second primary tumor occurs, the immune system broken by the first primary tumor has not been completely repaired and can not be triggered again to play its immune defense and other functions ${ }^{[7]}$. In recent years, a large number of literatures have reported that the prognosis of patients with metachronous multiple primary tumors is better than that of simultaneous multiple primary tumors ${ }^{[8-9]}$. The etiology of multiple primary tumors is not clear, which is generally considered to be the result of the joint action of multiple carcinogenic factors. To clarify the etiology of multiple primary tumors is very important to improve the prognosis of patients with lung cancer complicated with other primary tumors. After consulting relevant literature, it is speculated that the occurrence of multiple primary tumors may be related to the following factors: 1) genetic factors: it has been reported that the probability of multiple primary tumors in patients with family history of tumors is higher than those without family history of tumors ${ }^{[10]}$, which may be related to mismatch repair gene defects or chromosome dominant gene mutations ${ }^{[11-13] . ~ 2) ~ I m m u n e ~ f a c t o r s: ~ t h e ~ i m m u n e ~ s u r v e i l l a n c e, ~}$ immune defense and other functions of tumor patients are decreased, the sensitivity to carcinogenic factors is increased, and tumors are easy to reoccur ${ }^{[14-15]}$; 3) Use of chemotherapy drugs: a large number of animal experiments have confirmed that some anticancer drugs such as adriamycin and alkylating agents have different degrees of cancer promoting effects at the same time. Clinical studies have also shown that the incidence of the second tumor increases after chemotherapy for lymphoma ${ }^{[16]}$; 4) Radiotherapy: radiotherapy can increase the incidence of the second primary tumor, which may be related to the DNA damage, oncogene activation and tumor suppressor gene inactivation caused by 
radiotherapy; 5) The internal factors of the host include susceptibility, immune status, endocrine and embryonic development. Tumor patients may often cause the decline of the mechanism of the internal environment, reduce their adaptability to external and internal stimuli, easily accept the continuous stimulation of carcinogenic factors, and are prone to carcinogenesis at the same time ${ }^{\text {[17] }}$. Multiple cancers of female reproductive system may also be related to endocrine factors and embryonic development factors. The diagnosis of multiple cancers is difficult and easy to be confused with metastatic cancers. Therefore, special care should be taken in the diagnosis and relevant examinations should be improved to avoid missed diagnosis and delay treatment. So how did the patient develop canceration in the right maxillary sinus ten years later? We speculate that it may be related to the canceration of cells caused by multiple radiotherapy and chemotherapy.

Another concern of this case is whether the lung tumor is primary or metastatic at the second visit. In most cases, the primary lung tumor is a single mass, while the lung metastasis is a multiple mass with rough boundary. However, there are still some atypical manifestations of lung metastasis, including single metastasis, burr or lobulation, and intratumoral vasodilation, It is difficult to distinguish atypical lung metastases from primary lung cancer by conventional imaging. Some factors restrict the accuracy of CT diagnosis. Referring to relevant literature, we know that energy spectrum CT can perform multi parameter imaging based on traditional CT imaging, and can perform energy spectrum characteristic analysis, which can effectively eliminate hardening artifacts and improve image quality. It shows advantages in tumor qualitative, grading and differential diagnosis ${ }^{[18-21]}$. Therefore, energy spectrum parameters and single energy images can be used to identify whether the nature of lung tumors is primary or metastatic, which has certain clinical value and improve the diagnostic accuracy. At present, energy spectrum CT has not been carried out in our hospital. Therefore, in order to exclude the lung tumor as the source of metastasis, the patient first removed the lung tumor, and the pathological result was lung adenocarcinoma, which further confirmed that the lung tumor was primary. As of today's follow-up, the surgical area of bilateral maxillary sinus recovered well without recurrence, so it is proved that the treatment scheme of this case is relatively reasonable.

The maxillary sinus is located in the middle of the face and hidden. Once malignant tumors occur, most of them are late lesions that have invaded the surrounding structures. Surgery is the main treatment for maxillary sinus malignant tumors. The face collapse, orbital floor defect and hard palate defect left after maxillary resection have a great impact on the quality of life of patients. Local repair methods include local pedicled temporalis muscle flap, vascularized free tissue flap, prosthesis, etc. local pedicled temporalis muscle flap can repair the defects of hard palate and orbital floor ${ }^{[22]}$. Its advantage is that it can carry out functional reconstruction, its technical requirements are low, and the lesion is in the same operation area, with short operation time, rapid recovery of patients and low economic burden. Therefore, it is widely used at present.

At present, the incidence rate of multiple cancers in China is increasing. However, many cases are still discussed by case reports, and there are few studies on large-scale epidemiology, diagnosis, treatment and pathogenesis. Therefore, clinicians should pay enough attention ${ }^{[23]}$. Secondly, how to quickly detect 
and identify multiple primary malignant tumors through existing medical means, and determine effective treatment methods for multiple primary malignant tumors through precision medicine are the research directions we should strive for at present. Moreover, for otorhinolaryngology, this case of isochronous multiple lung cancer in bilateral maxillary sinuses is very rare, which also suggests that we should closely follow up tumor patients in clinical treatment. In addition to the primary site, we should also strengthen the monitoring of radiotherapy operation area and easily metastatic organs (such as lung, bone, liver, etc.), increase the popularization of relevant knowledge and improve the patient's awareness of disease prevention, Change the bad lifestyle, further reduce the incidence of multiple primary cancer or early detection of cancer, and improve the quality of life of patients.

\section{Declarations}

\section{Availability of data and materials}

All data generated or analyzed during this study are included in the published article (and its supplementary information files).

\section{Ethics approval and consent to participate}

I certify that this manuscript is original and has not been published and will not be submitted elsewhere for publication while being considered by Diagnostic Pathology. And the study is not split up into several parts to increase the quantity of submissions and submitted to various journals or to one journal over time. No data have been fabricated or manipulated (including images) to support your conclusions. No data, text, or theories by others are presented as if they were our own.

The submission has been received explicitly from all co-authors. And authors whose names appear on the submission have contributed sufficiently to the scientific work and therefore share collective responsibility and accountability for the results.

\section{Consent for publication}

Written informed consent was obtained from each patient for publication of this report and any accompanying images.

\section{Availability of data and materials}

The datasets used or analysed during the current study are available from the corresponding author on reasonable request.

\section{Abbreviations}

MPC: multiple primary cancer;CT:computed tomography;MRI: Magnetic resonance imaging;SOM:Secretory otitis media

\section{Competing interests}


The authors declare that they have no competing interests.

\section{Funding}

This research did not receive any specific grant(s) from funding agencies in the public, commercial, or not-for-profit sectors.

\section{Authors contributions}

Yue Liu, Yan LIU, Xue Zhao and Xian Hai Guo participated in the histopathological evaluation, performed the literature review and drafted the manuscript. Yue Liu and Dan Yu conceived and designed the study, Dan Yu revised the manuscript for important intellectual content. All authors read and approved the final manuscript.

\section{Acknowledgements}

Thanks are due to Xue Zhao for assistance with collecting the imagings and to Dan Yu for valuable discussion.

\section{References}

[1]Bai Yanxia,Yan Liying,Chen Yangjing \et al.Clinical analysis of 43 cases with carcinomas in maxillary

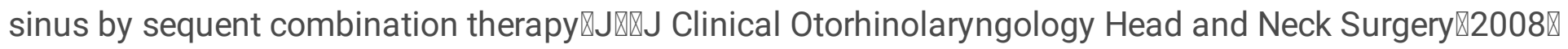
22(23) : 1073ه1075.

[2]Zhou Xing Qin,Qiu Xiao Jun,Zhao Hong Yu,et al.An analysis of treatment and prognosis of 17 cases with multiple primary cancer[J].Jiang su Med J,2014,40(12):1439-1440.

[3]Morton LM,Onel K,Curtis RE,et al.The rising incidenceof second cancers: patterns of occurrence and identification of risk factors for children and adults [J].Am Soc Clin Oncol Educ Book,2014(2014):e57e67.

[4]Zhao Ming Fang,Liu Yun Peng.Quadruple primary malignant tumor: a case report and literature review[J].J Chin Med Univ,2006,35 (3):325.

[5] Sylaja PN,Hill MD.Transient ischemic attacks-definition,risk prediction and urgent management\J『. Neurol India,2009,57ه3囚:252-256.

[6] Zeng YJ,Liu GF,Liu LP,et al. Anemia on admission increases the risk of mortality at 6 months and 1

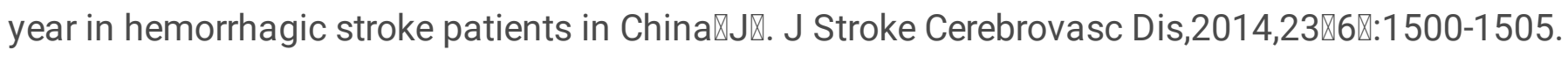

[7] Jiao F,Yao LJ,Zhou J,et al. Clinical features of multiple primary malignancies:a retrospective analysis

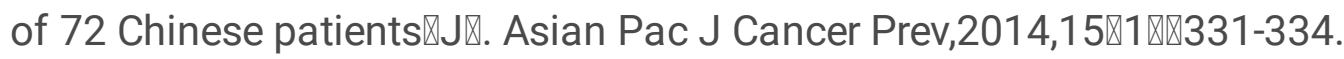


[8] Kim JH,Rha SY,Kim C,et al. Clinicopathologic features of metachronous or synchronous gastric cancer

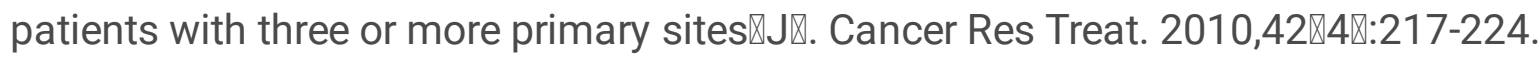

[9] Zeng QA,Qiu J,Zou R,et al. Clinical features and outcome of multiple primary malignancies involving

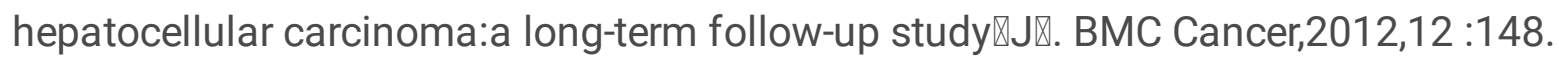

[10]Amer MH. Multiple neoplasms,single primaries,and patient survival[J]. Cancer Manag Res,2014,6 :119-134.

[11]Orlow I,Park BJ,Mujumdar U, et al. DNA damage and repair capacity in patients with lung

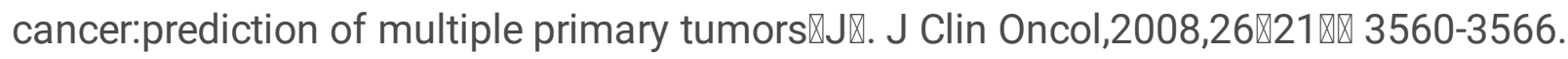

[12]Albright F,Teerlink C,Werner TL. Significant evidence for a heritable contribution to cancer predisposition:a review of cancer familiality by site区J囚. BMC Cancer,2012》 12:138.

[13]Win AK,Young JP,Lindor NM,et al. Colorectal and other cancer risks for carriers and noncarriers from families with a DNA mismatch repair gene mutation:a prospective cohort study[J].J Clin Oncol,2012,30 ख9::958-964.

[14]Trapani JA,Thia KY,Andrews M,et al.Human perforin mutations and susceptibility to multiple primary cancers[J]. Oncoimmunology,2013,2囚4凶囚e24185.

[15]Santangelo ML,Criscitiello C,Renda A,et al. Immunosuppression and Multiple Primary Malignancies in Kidney-Transplanted Patients:A Single-Institute Study $\mathbb{J} /$ OL】. http://www.hindawi.com/journals/bmri/2015/183523.

[16]Van Eggermond AM,Schaapveld M,Lugtenburg PJ,et al. Risk of multiple primary malignancies following treatment of Hodgkin lymphoma[J]. Blood,2014,124(3)『319-327.

[17]Mahmood S,Vu K,Tai P,et al. Radiation-induced Second Malignancies[J]. Anticancer Res,2015,35 $\llbracket 4 \rrbracket: 2431-2434$.

[18]Chen Ke Min,Lin Xiao Zhu.Clinical application, research value and Prospect of CT energy spectrum imaging[J].Modern Practical Medicine,2011,23( 8) : 841-844.

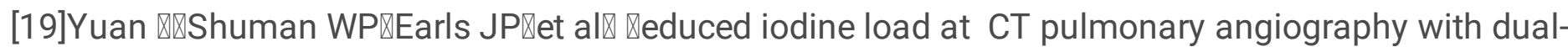
energy monochromatic imaging: comparison with standard CT pulmonary angiography-a prospective

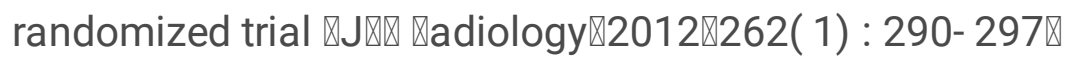

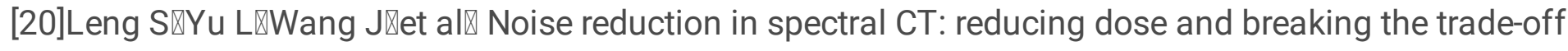

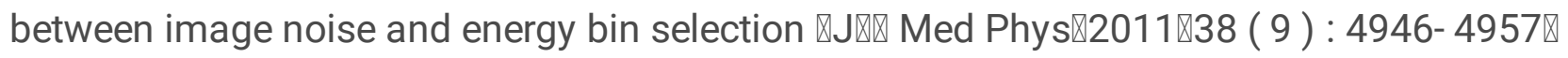

[21]Hu Xing He.Application Value of Gem Energy Spectrum CT for Screening of Early Lung Cancer[J].Clinical Medical Equipment,2011,32(12) : 64-65,78囚 
[22]Zhong Qi,Huang Zhi Gang,Fang Ju Gao,et al.Simultaneous repairing defects of orbital floor and palate with the modified temporalis muscle flap after the maxillectomy.Chin $\mathrm{J}$ Otorhinolaryngol Head Neck Surg,2016,51(9): 671-674.

[23]Sun Chuan Zheng,Chen Fu Jin,Li Xiao Jiang,et al.Clinical analysis of multiple primary carcinoma[J]Chin Arch Otolaryngol Head Neck Surg,2008(9):497-499.

\section{Figures}
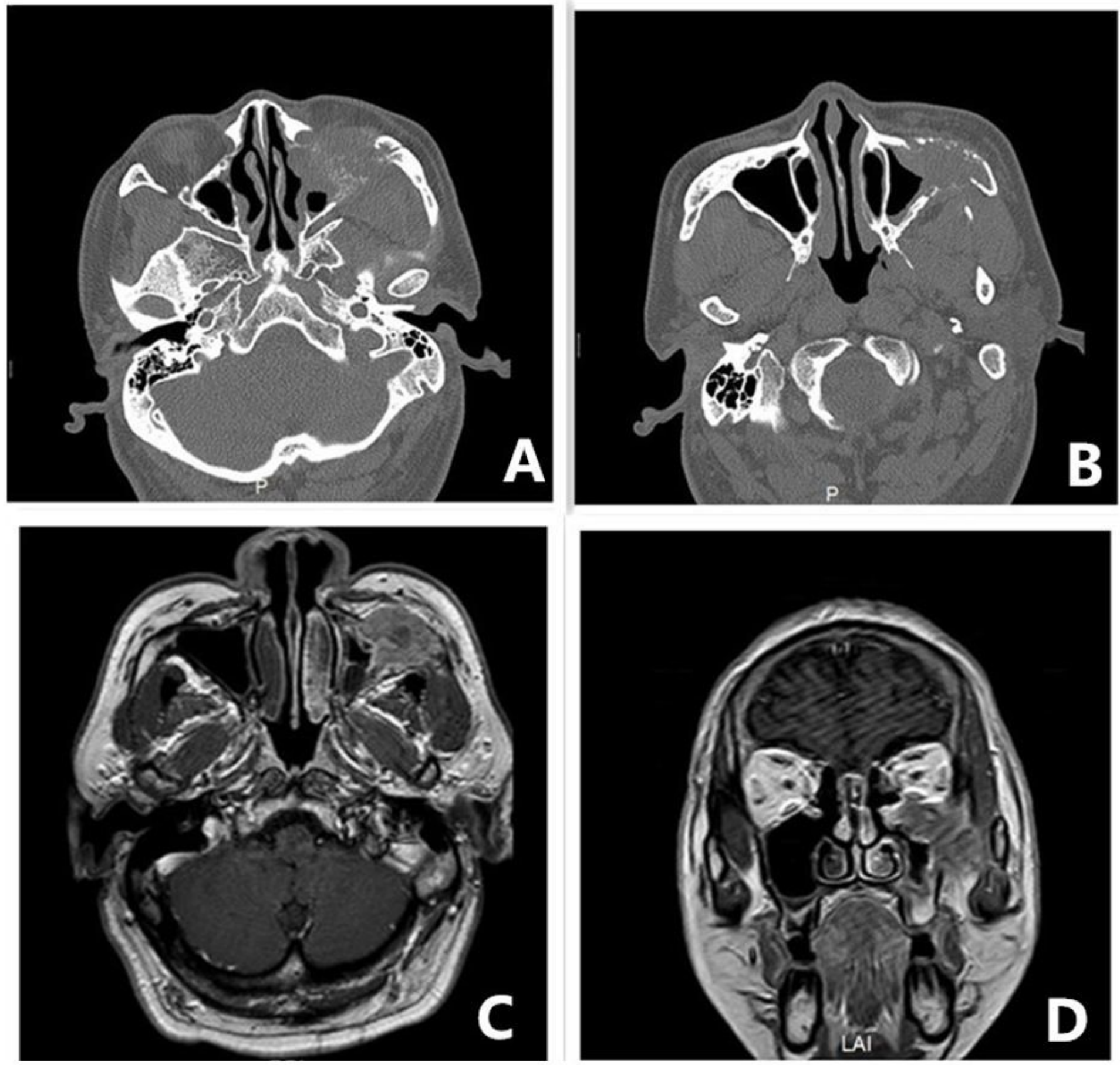

Figure 1 
$A, B$ : part of the left maxillary bone is destroyed and irregular soft tissue shadow can be seen next to it. Figure C,D: the abnormal signal of the left maxillary sinus shows mild to moderate enhancement,the left maxillary sinus cavity is enlarged and the signs of bone destruction appear on the lateral wall of the left maxillary sinus.
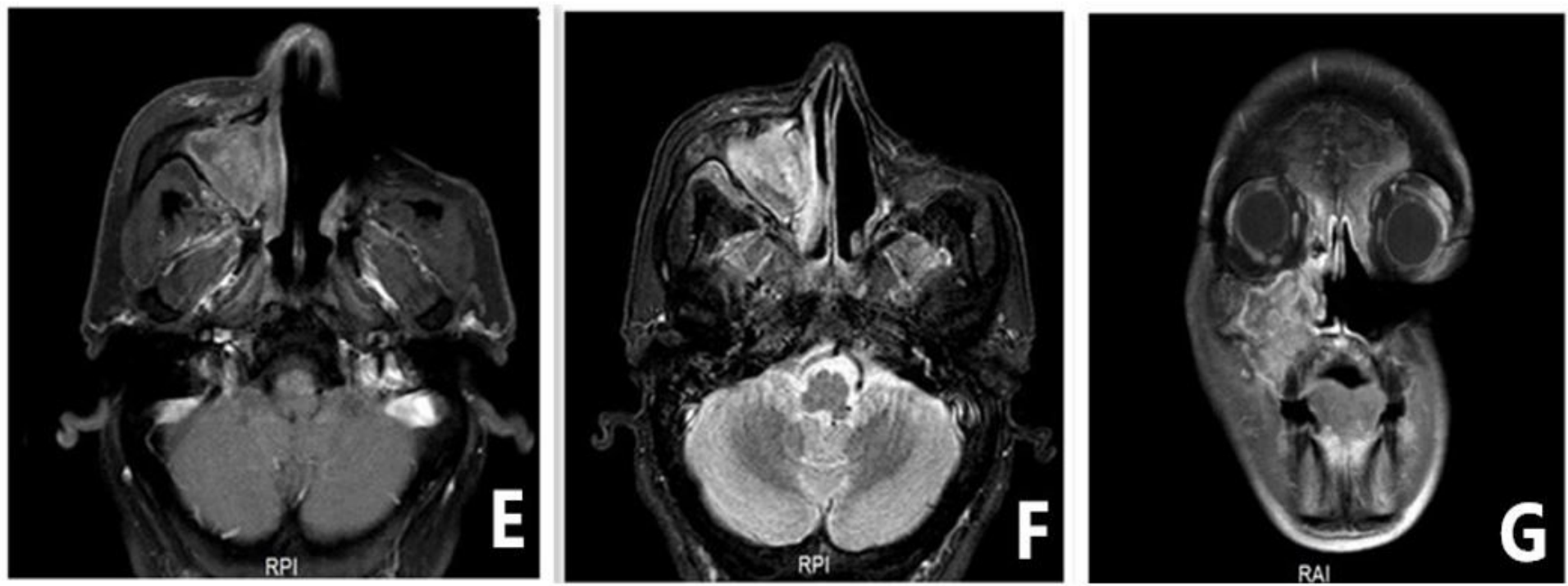

Figure 2

E, F, G: absence of bone in most of the left maxilla, local zygomatic arch, middle and lower turbinate, and discontinuous signal in some ethmoid trabeculae. Slightly longer T1 and slightly longer T2 signal shadow in the right maxillary sinus, bilateral frontal sinus, ethmoid sinus and sphenoid sinus. The enhancement scan showed uneven enhancement. 


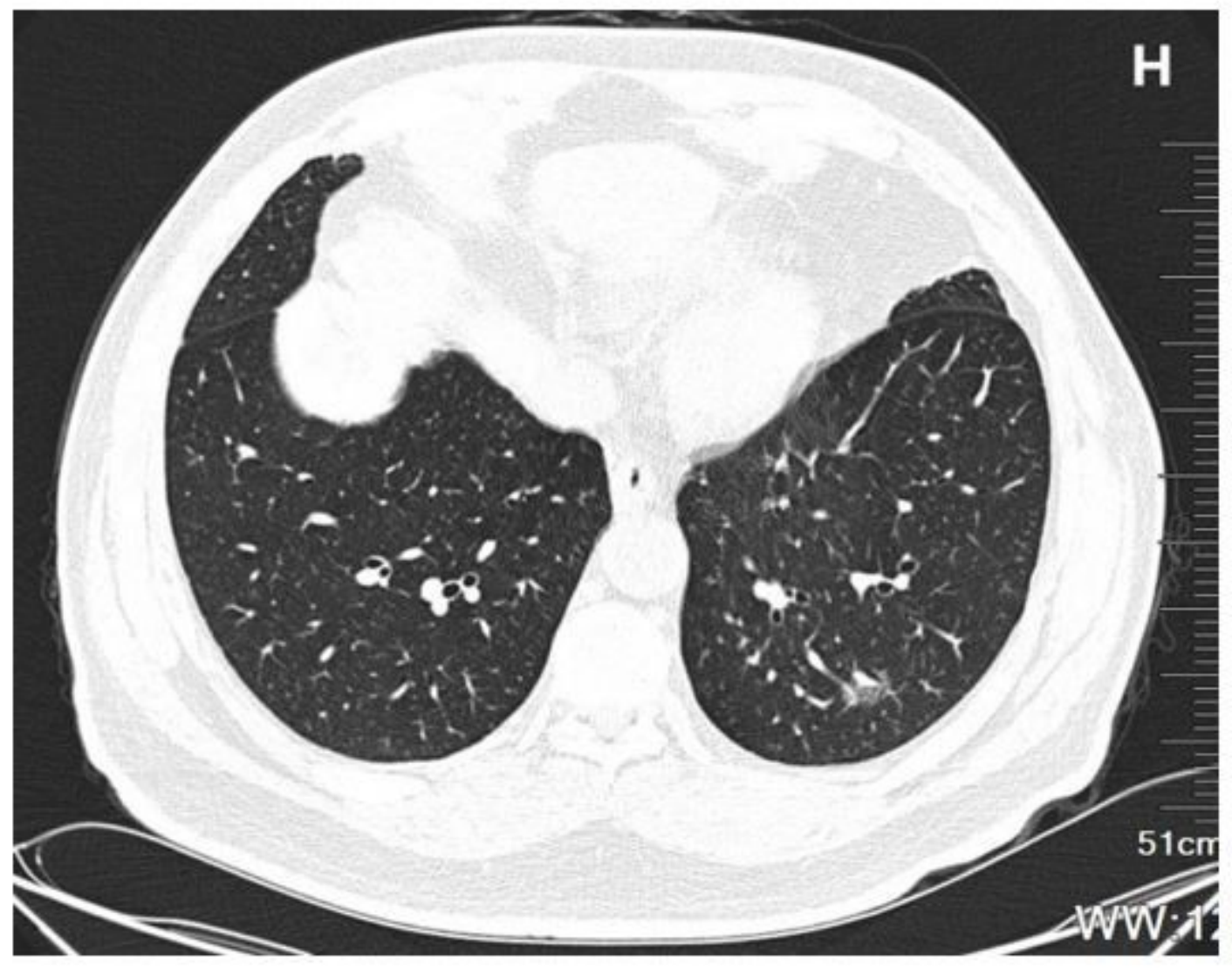

Figure 3

$\mathrm{H}$ : ground glass nodule shadow is seen in the lower lobe of the left lung (im207), with a size of about $17 \mathrm{~mm} * 11 \mathrm{~mm}$.
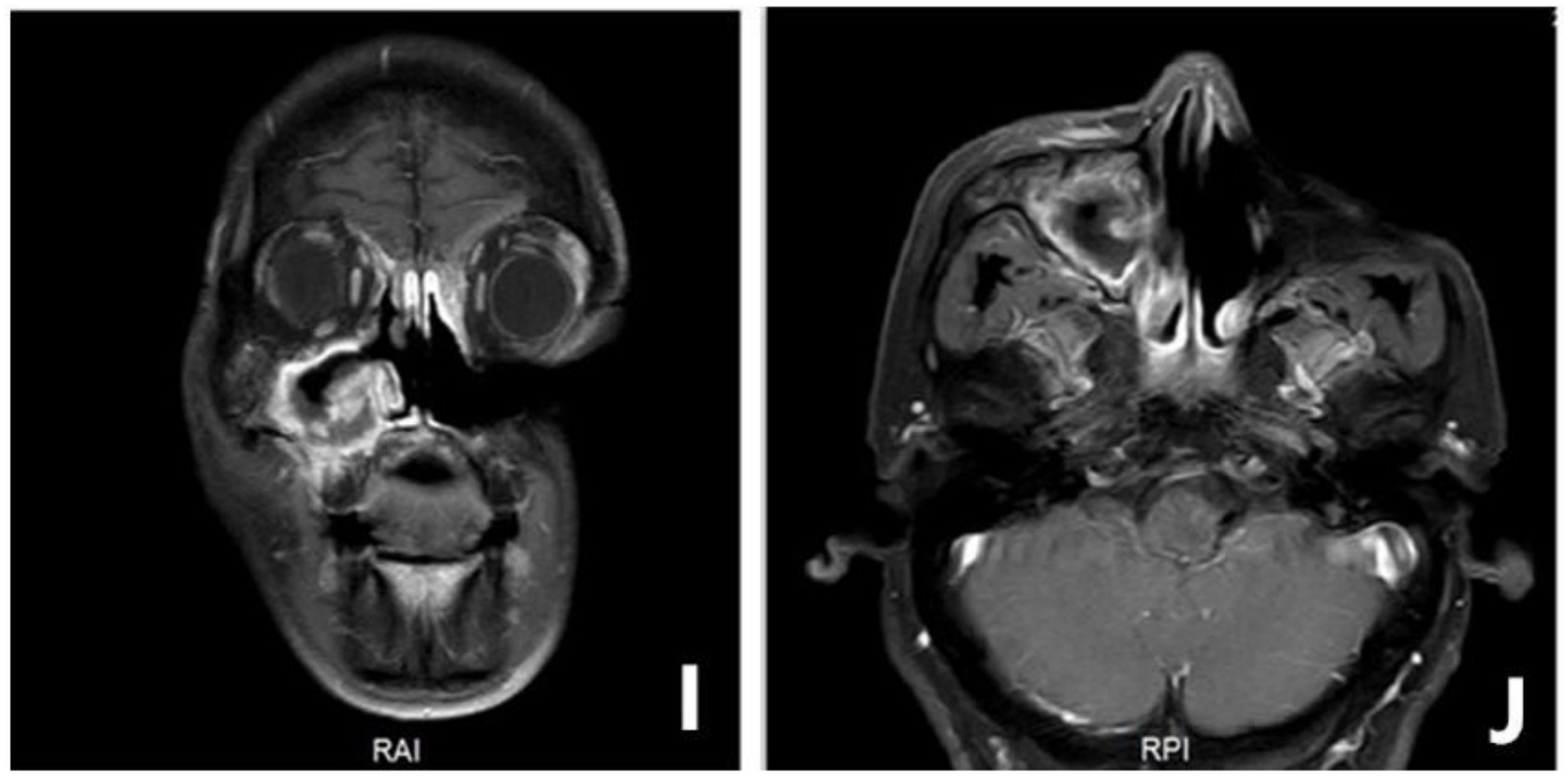

Figure 4 
$\mathbb{} \mathbb{} \mathrm{J}$ : slightly longer $\mathrm{T} 1$ and slightly longer $\mathrm{T} 2$ signal shadow in the right maxillary sinus, bilateral frontal sinus, ethmoid sinus and sphenoid sinus. The enhancement scan showed uneven enhancement, which was smaller than that in Figure $E, F$ and $G$.
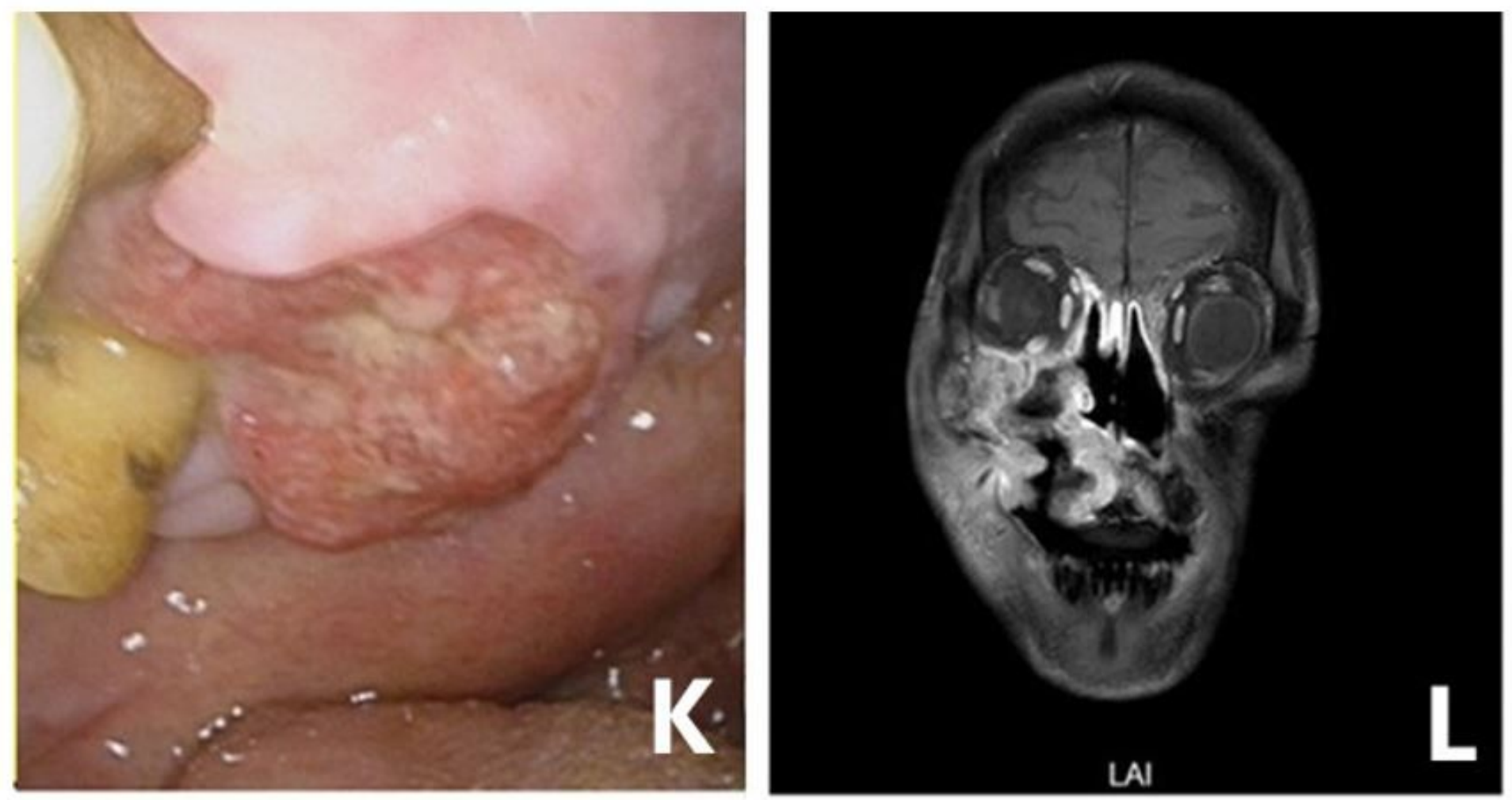

\section{Figure 5}

$\mathrm{K}$ : ulcerative neoplasia can be seen in the right hard palate. Figure L: the morphology of the right maxillary sinus is irregular. Slightly longer T1 and slightly longer T2 signals can be seen in each sinus wall and sinostomal nasal tract complex. The sinus cavity is narrowed. DWI shows slightly higher signals. The enhancement scan shows uneven enhancement. The focus downward involves the right side of the maxillary alveolar bone and the hard palate. Patchy long T2 signals can be seen in the right temporal muscle and maxillofacial soft tissue. 


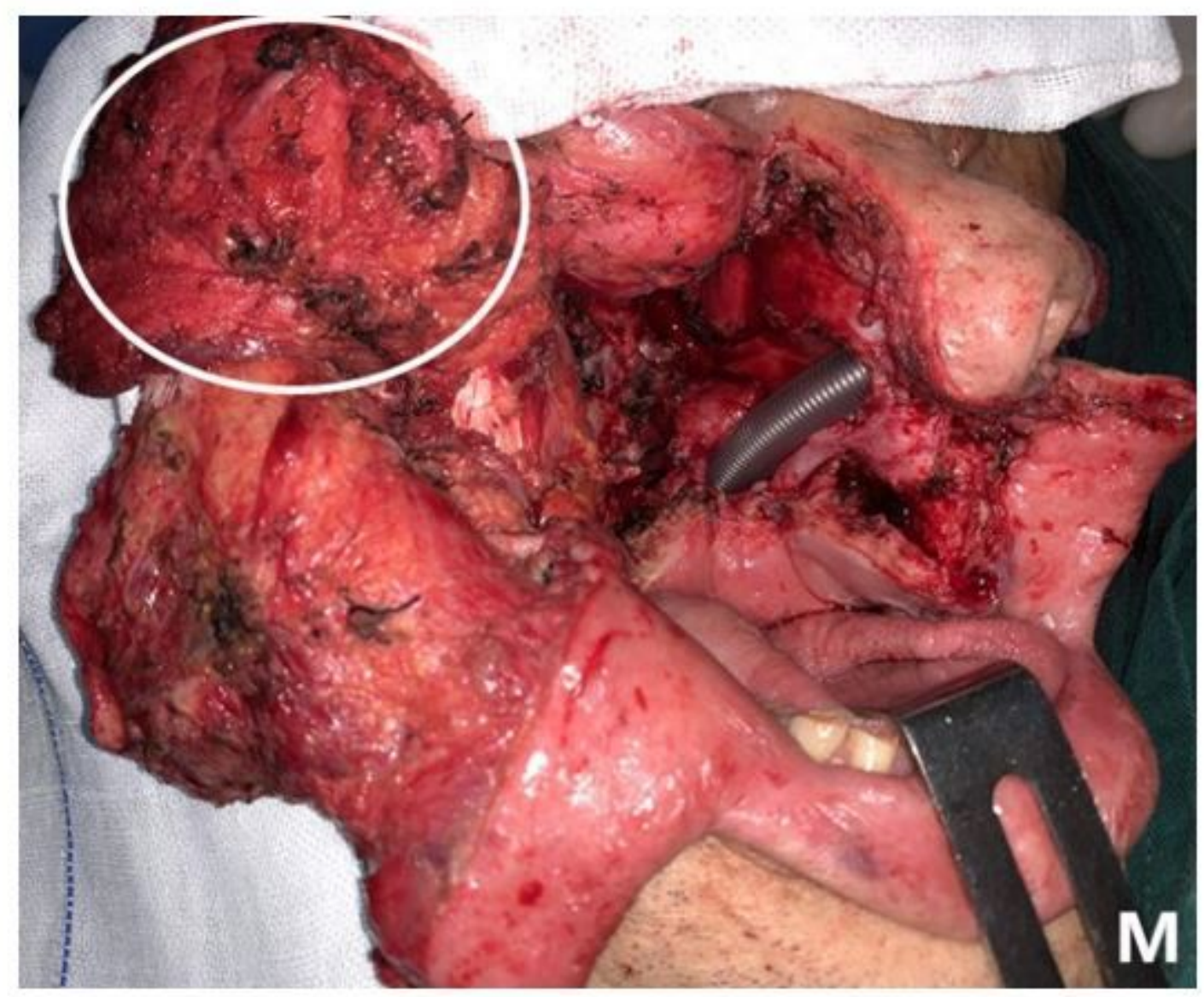

Figure 6

$\mathrm{M} \llbracket$ circle shows the pedicled temporalis muscle flap. 


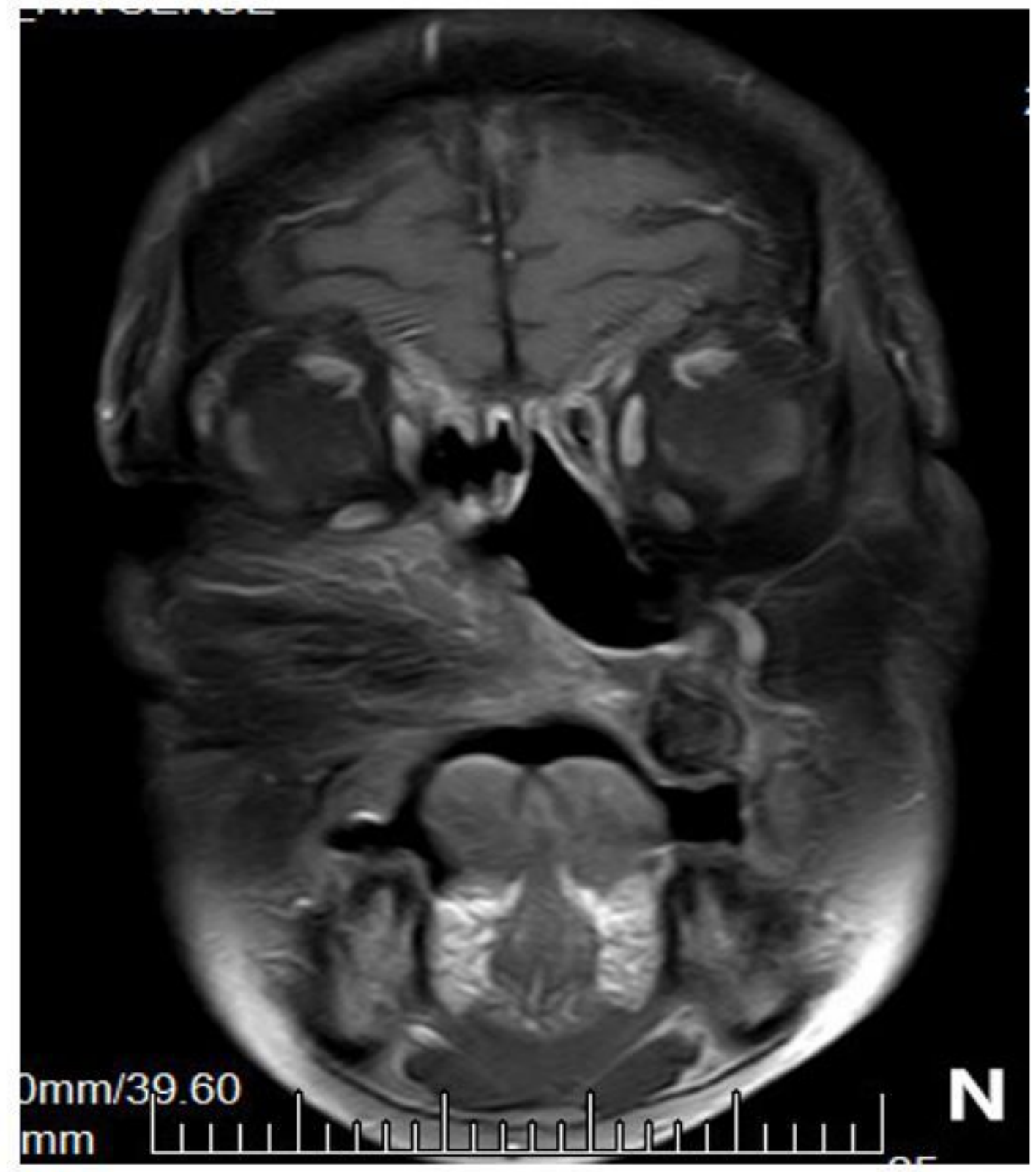

Figure 7

$\mathrm{N}$ : changes of bilateral maxillary sinus after operation, no abnormal signal shadow is found.

\section{Supplementary Files}

This is a list of supplementary files associated with this preprint. Click to download.

- carechecklist.jpg 